\title{
Computer programming for generating visual stimuli
}

\author{
Farhan BukHari and Daniel D. KuRYlo \\ Brooklyn College, CUNY, Brooklyn, New York
}

\begin{abstract}
Critical to vision research is the generation of visual displays with precise control over stimulus metrics. Generating stimuli often requires adapting commercial software or developing specialized software for specific research applications. In order to facilitate this process, we give here an overview that allows nonexpert users to generate and customize stimuli for vision research. We first give a review of relevant hardware and software considerations, to allow the selection of display hardware, operating system, programming language, and graphics packages most appropriate for specific research applications. We then describe the framework of a generic computer program that can be adapted for use with a broad range of experimental applications. Stimuli are generated in the context of trial events, allowing the display of text messages, the monitoring of subject responses and reaction times, and the inclusion of contingency algorithms. This approach allows direct control and management of computer-generated visual stimuli while utilizing the full capabilities of modern hardware and software systems. The flowchart and source code for the stimulus-generating program may be downloaded from www.psychonomic.org/archive.
\end{abstract}

We give here an overview of practical considerations for generating customized graphics for vision research. We include a brief overview of hardware considerations, followed by a discussion of programming strategies that allow specification of stimuli while utilizing memory and processing capacities of modern graphics hardware. We also include a discussion of suitable application programming interfaces (APIs) in the Windows operating system. We make recommendations for software implementations, but we also provide information needed for selecting an approach suitable for specific needs. Finally, we provide specific code that can be used as a framework for generating visual stimuli and controlling trial events. Programming examples address graphics control, animation techniques, color palettes, and timing techniques. We provide programming details to allow conversion of DOS-based VGA graphics to Win 32 programs, or for generating new programs for a broad range of research applications.

Commercially available software for application in vision research (see a representative list in Table 1) typically provides a library of common stimuli that may be modified within a framework. Such software does not provide the flexibility to generate unique stimuli required for novel and evolving research areas. Therefore, customized programming for generating stimuli within the context of specific research applications is needed. Programming examples provided here are designed for researchers with basic experience in computer programming. Programming examples describe the generation of simple stimuli, whereas the general approach may be used to generate complex and dynamic stimuli.

\section{VGA Graphics}

The VGA graphics standard was used extensively during the 1980s and early 1990s, and was applied in the generation of stimuli for behavioral research (Bokhorst, 1995; Diesch, 1994; Paredes, Miller, \& Creeger, 1990). Computer hardware at that time did not have the extensive computing power available today. The VGA standard was suitable because it offered limited resolution and colors, thereby minimizing computation and memory requirements. Also, DOS encouraged programmers to access hardware directly rather than through abstraction layers. However, DOS is a nonpreemptive system, restricted to relatively low spatial resolution, limited color depth, and limited access to the abundant graphics memory now available. With the newer, 32-bit flat memory model that offers protection to programs, programmers are free to use up to $4 \mathrm{~GB}$ of memory space. Current personal computers also typically contain $512 \mathrm{MB}$ to $1 \mathrm{~GB}$ of main memory, with graphics adapters containing up to $256 \mathrm{MB}$ of memory with a separate graphics processor. Therefore, approaches other than programming for VGA graphics are required to utilize current graphics capabilities.

\section{Monitors}

Older monitors were limited by a spatial resolution of $640 \times 480$ pixels (VGA standard). Although mainstream computing uses $800 \times 600$ pixels (SVGA standard) or $1,024 \times 768$ pixels (XGA standard), current technology allows up to 7,680 $\times 4,800$ pixels (WHUXGA, wide hexadecatuple ultra extended graphics array). Common monitors have resolutions ranging from $1,600 \times$ 
Table 1

Commercial Software for Stimulus Generation

\begin{tabular}{ll}
\hline \multicolumn{1}{c}{ Company/Product } & \multicolumn{1}{c}{ URL } \\
\hline ActiveStim & www.activestim.com \\
CRS Visual Stimulus Generators & www.crsitd.com/catalog/visage/index.html \\
Morphonome Image Psychophysics & www.ski.org/CWTyler_lab/General/morph.html \\
Vision Egg & www.visionegg.org \\
VPixx Technologies & www.vpixx.com \\
\hline
\end{tabular}

1,200 pixels (UXGA standard) for normal screens to $1,920 \times 1,200$ pixels for wide screens. Also, because video adapters increasingly support digital monitors, it is important to have a monitor that accepts the digital video interface (DVI). When selecting a monitor, a balance must be struck between the desired resolution and the refresh rate. Consideration should also be made of monitor calibration, to maintain control and consistency of output (Olds, Cowen, \& Jolicour, 1999). Samples of monitors are listed in Table 2.

LCD monitors are less desirable for vision research. LCD monitors provide less of a range of refresh rates, color, and built-in contrast, and the image quality is reduced for resolutions outside of the native resolution for which the monitor was designed. Also, viewing angle has more impact on image quality for LCD monitors than for CRTs.

\section{Video Adapters}

Newer technologies in video adapters have recently been introduced, such as scalable link interface (SLI) by NVIDIA, the PCI Express bus, and the DVI port. SLI technology allows two video cards to be connected to the computer, with the second video card sharing the computing load of the first. The cards are connected by means of a printed circuit board, which allows them to communicate directly with each other. Software drivers control the workload between the cards, either by splitting each frame's computation between each card, or by assigning alternate frames to the cards. Once all computations are done, the output is channeled to the primary video card, to which the monitor is connected. This technology speeds graphics by roughly a factor of two. ATI has also released a competing technology known as the CrossFire.

PCI Express, although not a video card technology, serves as a step above the older technology. PCI Express is a slot on the motherboard intended to replace all existing expansion slots. In its first phase, PCI Express was able to transfer data up to $2.5 \mathrm{gB} / \mathrm{sec}$, which is faster than the accelerated graphics port (AGP) slot used by current video adapters. It is now common to find PCI Express $16 \mathrm{x}$ slots, which can theoretically transfer data at nearly $4 \mathrm{~GB} / \mathrm{sec}$.

Although PCI Express and SLI technology are designed to generate and transport graphics at high speeds, DVI enables transport accuracy. DVI contains the digital output of graphics cards rather than the analog output required for older monitors. Digital systems allow less interference than analog systems, and since each pixel's value is transmitted digitally, colors are displayed exactly as specified, eliminating discrepancy among monitors. DVI is also backward compatible with older monitors through the use of an adapter.
Another consideration is that manufacturers customize different families of video adapters for different purposes. Adapters are designed for gaming, professional use, or common desktop use. For example, video adapters for professional use have extensive computing power, whereas gaming adapters emphasize graphics output. On a gaming adapter, there may be several pipelines to speed delivery, but limited computing power, with a reliance on software to prerender graphics. Professional-level adapters contain more computing power to render graphics, which can then be saved and used later. For the purposes of vision research, graphics cards designed for gamers are recommended. Some examples of graphics adapters are provided in Table 3.

\section{Operating System}

Although the graphical user interface used by Macintosh at one time allowed greater control of graphics, Windows operating systems, particularly with the introduction of Windows 95, allow a similar level of access to video adapters. Silicon Graphics had also been making advances in graphics around the time that Macintosh computers were introducing the graphical user interface. However, in their initial years, most of their code was proprietary. When the revised edition of the API known as the OpenGL was released, cross-platform graphics became possible.

Table 2

\begin{tabular}{lll}
\multicolumn{3}{c}{ Table 2 } \\
\hline \multicolumn{1}{c}{ Brand } & \multicolumn{1}{c}{ Model } & \multicolumn{1}{c}{ Resolution } \\
\hline Seamless Monitor & Radius320 & $4,800 \times 1,200$ \\
ViewSonic & G225fB & $2,048 \times 1,536$ \\
Planar & SD2020 & $1,600 \times 1,200$ \\
Planar & SD2320 & $1,920 \times 1,200$ \\
Sharp & PN-455 & $1,920 \times 1,080$ \\
ViewSonic & E90fB & $1,792 \times 1,344$ \\
IBM & ThinkVision C220p & $2,048 \times 1,536$ \\
Conrac & 6028ID & $2,048 \times 2,048$ \\
NEC-Mitsubishi & Diamond Pro 2070 & $2,048 \times 1,536$ \\
ViewSonic & VP2330wb & $1,920 \times 1,200$ \\
ViewSonic & VX2035wm & $1,680 \times 1,050$ \\
ViewSonic & Q191wb & $1,440 \times 900$ \\
\hline
\end{tabular}

Table 3

Video Card Resources

\begin{tabular}{llcc}
\hline Brand & \multicolumn{1}{c}{ Model } & Specifications & Memory \\
\hline ATI & All-in-wonder x800 & $2,048 \times 1,536$ & $256 \mathrm{MB}$ \\
3D Labs & Wildcat VP990 Pro & $2,048 \times 2,048$ & $512 \mathrm{MB}$ \\
3D Labs & Wildcat Realizm 200 & $3,840 \times 2,400$ & $512 \mathrm{MB}$ \\
3D Labs & Wildcat Realizm 800 & $3,840 \times 2,400$ & $640 \mathrm{MB}$ \\
NVIDIA & GeForce 6800 GT & $2,048 \times 1,536$ & $256 \mathrm{MB}$ \\
& & SLI Ready & \\
NVIDIA & Quadro NVS 280 & $2,048 \times 1,536$ & $64 \mathrm{MB}$ \\
\hline
\end{tabular}


Mainstream operating systems are 32 bits wide, and theoretically allow access to $4 \mathrm{~GB}$ of memory. Certain 64-bit operating systems are also available, but require a 64-bit processor, and most of them are still in the development stages. Of the 32-bit operating systems that are available, Linux, Unix, OS X, and Windows are the most popular. Until recently, OS X was not compatible with the i86 architecture, and has not been widely used. Also, Macintosh hardware is proprietary and leaves little choice for users. Linux and Unix have a steep learning curve, because of their uniqueness, and are not designed as graphics systems. For these reasons, the widely used and widely supported Windows operating system (Petzold, 1998) is recommended.

\section{Graphics Implementation}

Several software packages and languages are available for graphics implementation, each with advantages and disadvantages for generating visual stimuli (Abrash, 1997). We discuss the most popular packages here.

VGA with assembly language. One approach is to use VGA graphics with assembly language, to gain greater control over graphics capabilities. Assembly language is the lowest-level programming language, with a one-toone correspondence with machine code. Compared with compiled code, assembly allows extremely fast execution and conserves memory. However, assembly code is processor specific and therefore not portable among different architectures. Although it is possible to interface assembly with libraries written for other languages, calling compiler-generated code by handwritten assembly does not constitute an advantage. Writing custom graphics from scratch in assembly would be tedious and would still be limited by the VGA standard. Also, with the capabilities of modern computers, speed and memory conservation are less important. Furthermore, modern compilers are much better at optimizing high-level code in comparison with what could typically be produced from scratch.

Java and Java2D. Java and its clones, such as Visual $\mathrm{J++}$ and Visual J\# (Sierra \& Bates, 2002), are high-level languages similar to a mixture of $\mathrm{C}$ and $\mathrm{C}+$ that are made portable by compiling into an intermediary code, known as "bytecode." Java and its clones include a large number of precompiled packages. Despite its advantages, Java is less desirable for vision research because Java is an interpreted language, which runs much slower than compiled code. Also, Java is not designed for high-performance code that may be required to specify stimulus metrics. Native interface can be used to work with the operating system's APIs, but the code will still be slow and tedious at best.

Vector graphics. Vector graphics are described using mathematical equations, and offer unlimited zooming without compromising the image quality. Two other approaches are to use either scalar vector graphics (SVG) or Asymptote. SVG is a vector graphics package that is programmed in extensible markup language (XML). XML is similar to hypertext markup language (HTML), the language used for most Web pages. XML, being a data description language, is parsed, and therefore requires significant overhead, reducing control over timing. Asymptote, unlike SVG, is a programming language itself and is able to generate graphics in the form of Adobe PostScript or Portable Document Format (PDF). Both packages require a third-party application, such as Ghostview, Adobe Acrobat, or a Web browser, to show their output, which is not convenient for experimental control.

OpenGL. OpenGL is among the most popular graphics packages. Function calls accompanying this package are meticulously detailed, and allow applications as complex as commercial games. OpenGL has been an evolving specification. Although the newer versions are backward compatible, not all computers have compatible hardware or drivers. Also, vendors are not bound to support all the OpenGL functions in their hardware or drivers, which may result in the entry points for the newer functions not being found. Additionally, not all Windows compilers contain header files and libraries for OpenGL. In those cases, the files must be updated manually. Although OpenGL is common and is used as a standard, it requires relatively high-end computers to render graphics smoothly, and many older computers are not compatible with it. Also, such an extensive package may exceed most graphics needs for vision research.

DirectX. Some of the problems associated with OpenGL are resolved by the DirectX graphics package, which comes standard with the Windows operating system. DirectX is composed of several components, including DirectDraw, which is suitable for graphics required for visual research. However, Direct $X$ is similarly complex as is OpenGL; unlike OpenGL, it is not available for operating systems other than Windows.

GDI. The graphical device interface (GDI) is a layer of abstraction consisting of several functions included with all versions of Windows as gdi32.dll. In Windows $\mathrm{XP}$, the updated version is included as gdiplus.dll. GDI handles all the graphics needs of the Windows operating system. With respect to the needs of vision research, GDI allows basic graphics programming, is relatively easy to program, and works on all computers running Win32. Programs using GDI are compiled and not interpreted, and run faster than interpreted programs. Programs are also $32 \mathrm{bit}$, and can therefore take advantage of the flat memory model. Because the entire package is in a dynamic link library, any programming language compatible with dynamic link libraries can take advantage of it. In comparison with OpenGL or DirectX, GDI is designed for more primitive graphics. GDI is recommended for vision research because it is able to handle basic graphics without a significant overhead or complex function calls, and without adding unneeded dependencies.

Some disadvantages of GDI are that it is not meant for 3-D graphics, or highly complex stimuli. Furthermore, it offers no built-in support for creating real-time animation. Texturing and lighting are also produced less effectively with GDI.

\section{Programming Language}

The languages we considered for programming the GDI were the Microsoft Managed Visual Studio .NET Framework, $\mathrm{C}$, and $\mathrm{C}+\mathrm{H}$.

Microsoft Managed Visual Studio .NET. Several languages may be compiled together and interfaced with each 
other seamlessly with .NET Framework. An advantage in using several languages for vision research is that some tasks are easier to accomplish with a certain language than with others. Compiling the code with .NET generates intermediary code known as Microsoft Intermediate Language (MSIL). When the applications need to execute, the MSIL code is compiled on the spot and run natively. Whereas it adds other features, too-such as allowing different levels of security, simplified packaging, and deployment-these features are less important to vision research. Also, a relatively powerful computer is required to run Microsoft Visual Studio .NET, and that is not always available.

$\mathbf{C}$ and $\mathbf{C + +}$. C creates tight, efficient code, and can be used to interface directly with Windows via the API. Extensive explanations of all the API function calls are available. $\mathrm{C}$ allows extreme flexibility while maintaining high-level properties, and generates executables that are typically small and efficient. C++ offers nearly all the benefits of $C$ except that the code is not always as compact and fast. In addition, wrapper functions associated with $\mathrm{C}++$, such as Microsoft Foundation Classes and ActiveX controls, which are provided to facilitate programming Windows applications, add another layer of indirection and therefore further reduce speed, and limit the programmer's options. All the complexity associated with $\mathrm{C}++$, wrapper classes, and object-oriented programming is not recommended for generating simple visual stimuli. We therefore recommend programming in $\mathrm{C}$, making direct API calls, working with the GDI, and using a development environment capable of generating 32-bit windowed executables. Some examples include unmanaged Microsoft Visual Studio .NET, and LCC-Win32.

\section{Programming Code and Application Structure}

Programming often involves finding the correct API. Specific APIs are used for monitor control, input/output, message channeling, timing, precomputing, and process control.APIs for each of these tasks are listed below, along with sections of code in which they are applied. Complete code for generating a stimulus within a trial is presented in the Appendix (www.psychonomic.org/archive).

WinMain. WinMain serves as the entry point of the program. This function can be used to change monitor settings, initialize windows, and loop through trial events. The primary responsibility of WinMain is to either enter the event loop and process incoming messages, or call other functions to do so. The following is an example of a basic WinMain function:

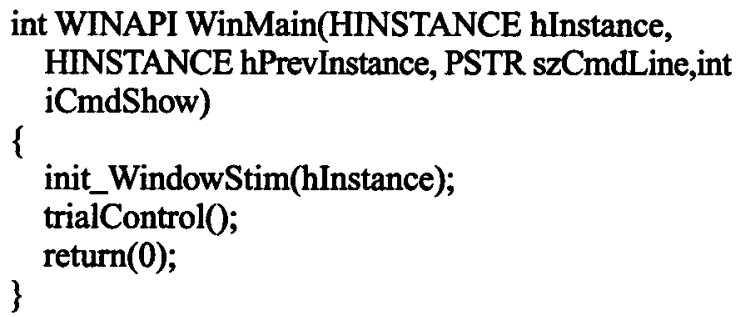

Setting spatial resolution and refresh rate. Screen resolution and refresh rate are set with the structure DEVMODE. The members dmPelsWidth, dmPelsHeight, and
dmDisplayFrequency represent the horizontal resolution, vertical resolution, and the vertical refresh rate, respectively. Once these fields have been initialized, the dmFields field must be initialized to indicate which fields of the structure are to be read and acted upon in ChangeDisplaySettings().

\section{DEVMODE devmode;}

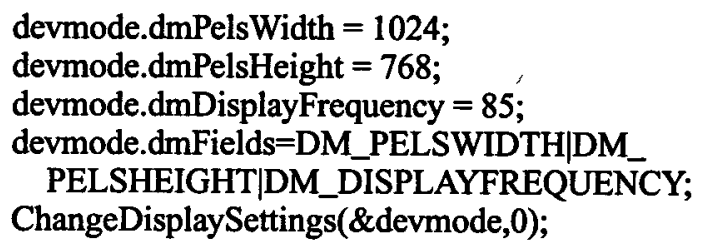

Setting color and luminance. Specifying color, or grayscale luminance, of objects and background can be done with RGBO, assigned to a variable of type COLORREF. Values of the three primary colors range from 0-255.
COLORREF color;
int red_value $=255$;
int green_value $=0$;
int blue_value $=255$;
color $=$ RGB(red_value, green_value, blue_value);// creates purple

Setting the stimulus window properties. The typical borders that accompany windows can be eliminated by specifying WS_POPUP as the window style attribute when creating the window. The maximum size of the screen can be obtained by calling GetSystemMetrics 0 . Other attributes, such as keeping the window on top, can be set by calling SetWindowPos(). The following example creates a borderless window that spans the entire screen and stays on top.

\section{fullScreenX = GetSystemMetrics(SM_CXSCREEN); fullScreenY $=$ GetSystemMetrics(SM_CYSCREEN); hwnd $=$ CreateWindow(...,...,WS_POPUP,0,0, fullScreenX,fullScreen $Y, \ldots, \ldots, \ldots$ ); \\ SetWindowPos(hwnd,HWND_TOPMOST, 0,0,0,0, SWP_NOMOVE | SWP_NOSIZE);}

Note that in this example, WS_POPUP will remove the close button from the title bar of the window (top right corner). Therefore, another mechanism is required to terminate the application conveniently.

Initializing the stimulus window. Stimuli are presented here in a devoted window. Creating a window and displaying it on the screen requires first initializing the WNDCLASS structure, registering it with the operating system using RegisterClass 0 , and then creating the window by calling CreateWindow(). Note that this code creates a window in the computer memory only, and does not display the window on the screen.

HWND hwndStim; WNDCLASS StimWindow;

$$
\begin{aligned}
& \text { void init_WindowStimulus(HINSTANCE hInstance) } \\
& \text { StimWindow.style=CS_HREDRAW | }
\end{aligned}
$$


CS_VREDRAW;

StimWindow.lpfnWndProc $=$ WndProcStim;

StimWindow.cbClsExtra $=0$;

StimWindow.cbWndExtra $=0$;

StimWindow.hInstance $=$ hInstance;

StimWindow.hIcon=LoadIcon(NULL,IDI_ APPLICATION);

StimWindow.hCursor=LoadCursor(NULL, IDC_ ARROW);

StimWindow.hbrBackground $=($ HBRUSH)

GetStockObject(RGB(100,100,100));

StimWindow.lpszMenuName=NULL;

StimWindow.lpszClassName="Stimulus_ Window";

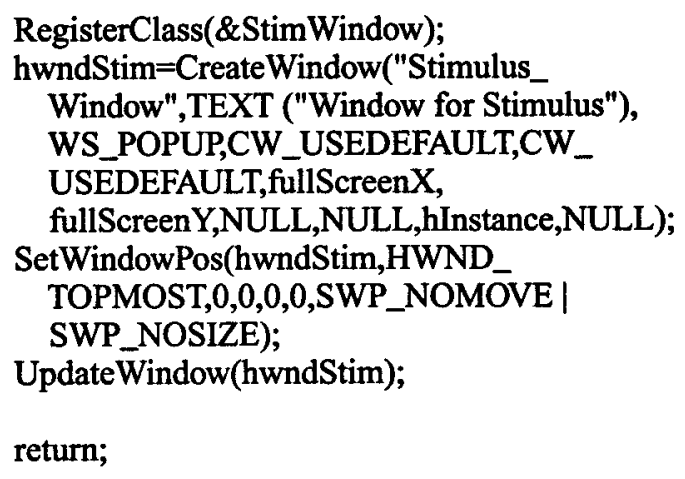

RegisterClass(\&StimWindow);

hwndStim=CreateWindow("Stimulus _ Window",TEXT ("Window for Stimulus"), WS_POPUP,CW_USEDEFAULT,CW_ USEDEFAULT,fullScreenX, fullScreenY,NULL,NULL,hInstance,NULL);

SetWindowPos(hwndStim,HWND_

TOPMOST,0,0,0,0,SWP_NOMOVE |

SWP_NOSIZE);

UpdateWindow(hwndStim);

return;

\}

Creating the stimulus. Stimuli are drawn on the window by calling a variety of GDI functions. GDI is used with a device context, which is a structure maintained by the operating system that holds the attributes of a particular output device. In order to send output to that device, the program must first obtain a handle to that device context using one of several functions, such as BeginPaint() and GetDCO.

GDI is also used to create or manipulate built-in objects and memory. The process for creating a bitmap in the memory is as follows:

(1) Get the handle to the stimulus window by calling GetDC0.

(2) Create a bitmap that is compatible with the stimulus window by calling CreateCompatibleBitmap 0 .

(3) Create a memory object that is compatible with the window by calling CreateCompatibleDC 0 .

(4) Select the bitmap into the memory device context by calling SelectObject(0. The bitmap can then be manipulated by manipulating the memory associated with it.

CreateCompatibleDC( $)$ creates a memory object that is compatible with a particular output device. This is useful because instead of manipulating the output device itself, this memory area can be used to generate the stimulus before sending it to the output device. Selecting the bitmap into this memory location designates which object is to be manipulated.

GDI also provides several convenient functions that can be used to draw a stimulus, such as Rectangle(), Ellipse(),
Arc0, LineTo0, Polygon(), and PolyBezier(). These functions are used here to draw the stimulus on the bitmap created in memory. The following code creates a bitmap in the computer's memory, paints the background gray, and then draws a square 20 pixels long at the coordinates $x$ and $y$.

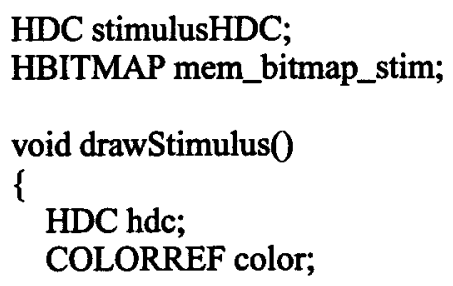

HDC stimulusHDC;

HBITMAP mem_bitmap_stim;

void drawStimulus 0

\{

HDC hdc;

COLORREF color;

hdc $=$ GetDC (hwndStim);

mem_bitmap_stim=CreateCompatibleBitmap

(hdc,fullScreenX,fullScreenY);

stimulusHDC $=$ CreateCompatibleDC $(0)$;

SelectObject(stimulusHDC,mem_bitmap_stim);

// set background

color $=$ RGB $(100,100,100)$;

SelectObject(stimulusHDC,CreateSolidBrush (color));

Rectangle(stimulusHDC, 0,0,fullScreenX, fullScreenY);

// build objects

color $=\mathrm{RGB}(255,255,255)$;

drawSquare(stimulusHDC, x, y, 20, color);

ReleaseDC(hwndStim,hdc);

return;

\}

void drawSquare(HDC frame, int left, int top, int size, COLORREF color)

\{

int $\mathrm{x}, \mathrm{y}$;

HBRUSH hbrush;

HPEN hpen;

hbrush $=$ CreateSolidBrush(color);

hpen= CreatePen(PS_NULL,0,RGB $(0,0,0))$;

SelectObject(frame,hbrush);

SelectObject(frame,hpen);

Rectangle(frame,left,top,left+size,top+size); return;

\}

Presenting the stimulus. Unlike DOS, Windows allows users to actively interact with the objects on the screen generating events. The operating system tracks these events and passes them to the active window in the form of messages. All Windows applications must have at least one message loop that receives these messages and dispatches them to the correct window handler, as specified by the lpfnwndProc field of the WNDCLASS struc- 
ture associated with that particular window. In order to make sure that the stimulus window handler receives these messages, this window has to be active. Once the messages are directed to the correct window handler, the stimulus can be manipulated by means of these messages.

The stimulus can now be displayed on the screen. In the code that follows, InvalidateRect 0 generates the WM_ PAINT message, which paints the stimulus on the window. ShowWindow0 is then used to display the painted window on the monitor.

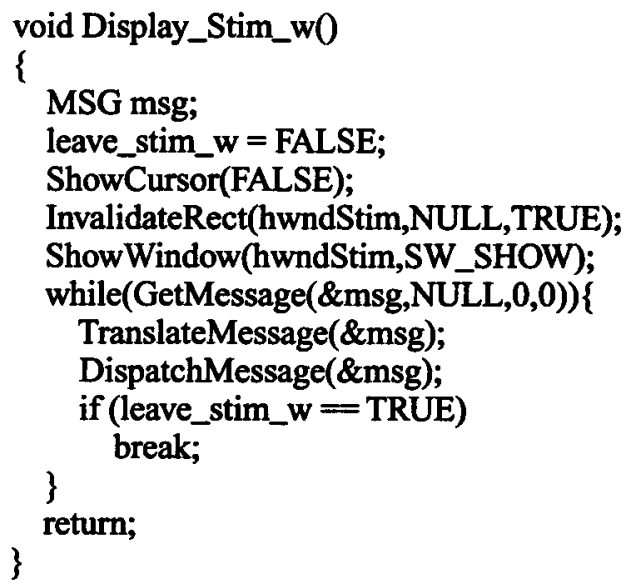

DispatchMessage $($ invokes $1 p f n W n d P r o c()$, a pointer to a window procedure function. With this function, messages that have significance for the stimulus are properly handled. This handling may range from receiving keyboard input and detecting mouse clicks to more complex processing such as the creation of stimuli. In this example, the message WM_PAINT invokes BitBlt0, which copies the bitmap in the memory to the device context of the stimulus window. The stimulus is thereby painted on the window.

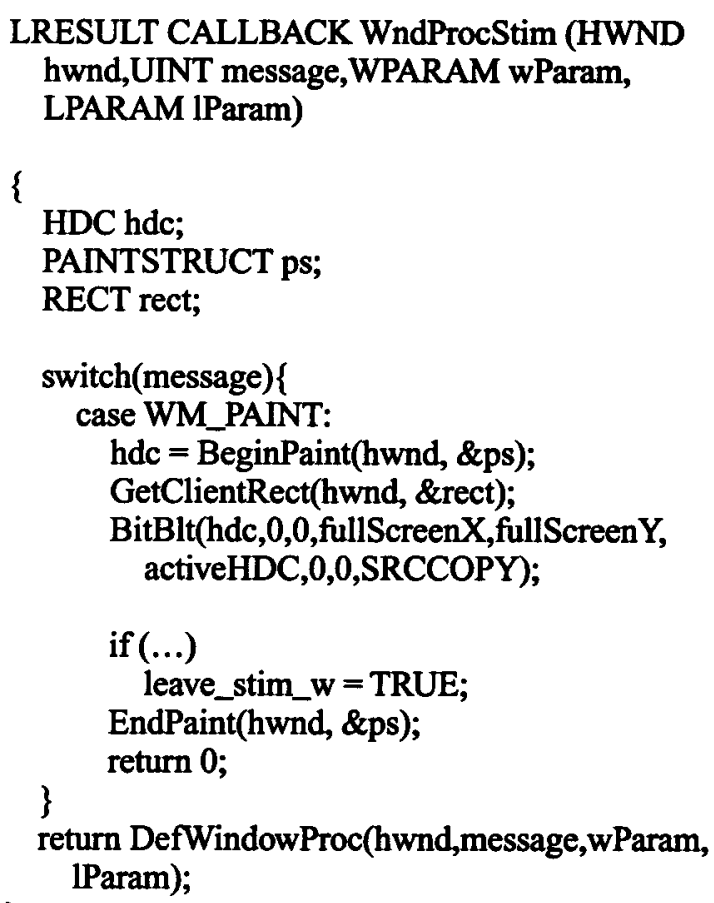

Controlling stimulus duration. Stimulus duration needs to be linked to the vertical refresh of the monitor, which specifies the temporal resolution of stimuli presentation. Vertical refresh is read through the fourth bit of Port 986, which may be done with the _inp0 function. However, because operating systems like Windows XP trap instructions that try to read directly from a port, the application permission level needs to first be adjusted. One way to accomplish this is to install a device driver called GiveIO.sys with an installer called LoadDrv.exe. This is a one-time installation, and both files are freely available on the Internet. This step is not required for Windows 95/98/ME.

Once the device driver has been installed, the following code can be used to alter the input/output (I/O) permission bitmap for a particular process.

\section{hfile=CreateFile("III.IIgiveio",GENERIC_READ,0, NULL,OPEN_EXISTING,FILE_ATTRIBUTE_ NORMAL,NULL); \\ CloseHandle(hfile);}

After this code has been executed, the vertical refresh pulse can be monitored by isolating the fourth bit of Port 986 , as follows:

value $=\_$inp(986)\&8;

Finishing a trial. The bitmap object and the device context must be deleted after they are used; otherwise a memory leak will result. To delete an object, call the DeleteObject() function and pass it the handle to the object to be deleted (created with CreateCompatibleBitmap()). To delete the device context, call DeleteDCO and pass it the handle to the device context (obtained with CreateCompatibleDC0).

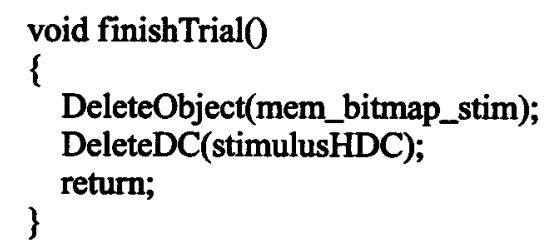

Presenting animation. Animation may be produced by a number of strategies. One approach is to precompute and store individual frames with separate handles. Later, the animation frames can be displayed by stepping through the handles sequentially. For generic use, each frame's handle is assigned to a general handle, which is used when displaying the stimulus. Use of a general handler also allows convenient inclusion of other types of displays, such as a fixation point, cue, and mask. In the following example, five stimulus frames are created in which the square moves down and right.

First, precompute each stimulus frame:

HDC stimulusHDC[5];

HBITMAP mem_bitmap_stim[5];

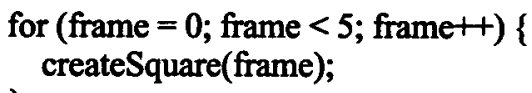




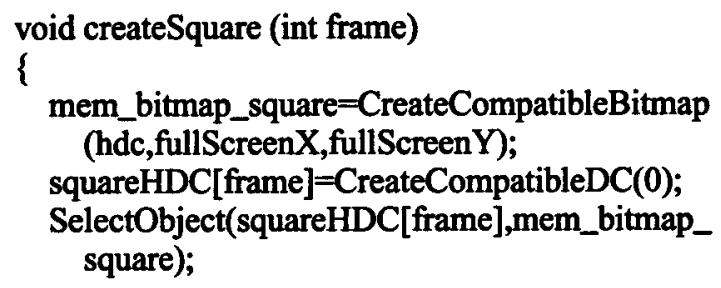

Now display each frame:

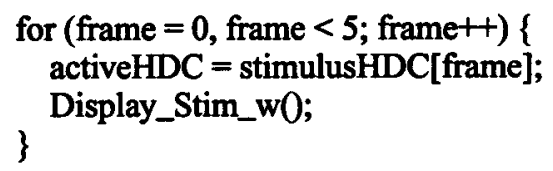

Note that precomputing frames may not be the optimal strategy for animation that involves simple changes, or one that requires a large number of frames. For these situations, other strategies should be used.

GDI is appropriate for basic animation. When working with bitmaps and GDI, stimulus frames may be saved in files. However, graphics containing high resolution, deep colors, or multiple frames will tax the memory significantly, since bitmaps do not employ compression algorithms. For such stimuli, OpenGL is better suited.

Another strategy is to calculate differences from frame to frame and update only changed areas. This procedure may be complicated when tracking overlapping textures.

Presenting picture images. An option other than building stimuli with graphics APIs is to call and display a previously stored picture image. The picture can be loaded into memory at any time during the program's execution, but it should be copied onto the window in WM_PAINT. In the following example, the bitmap file picture.bmp is displayed on the screen.

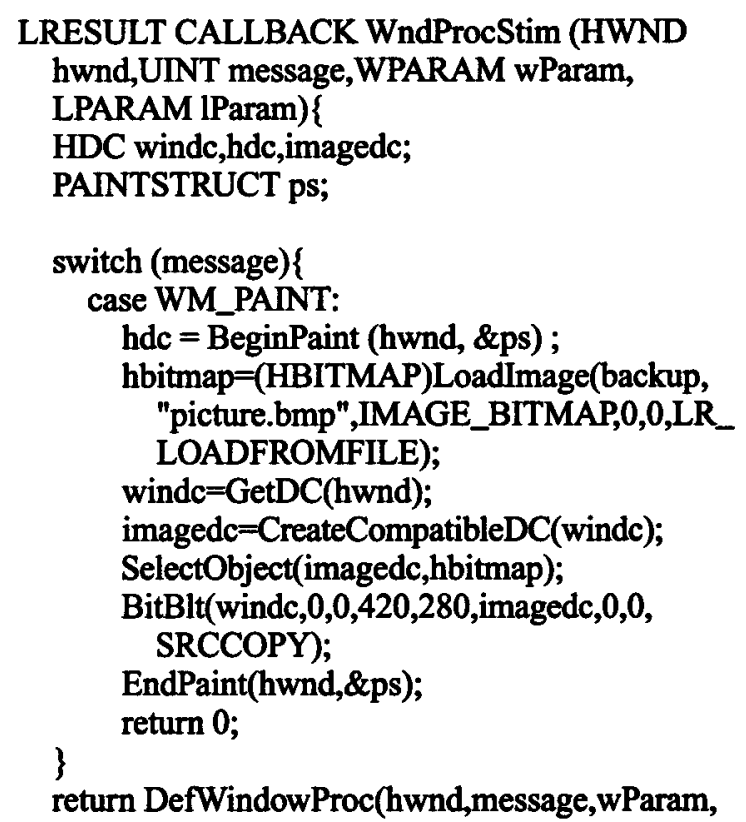

\section{IParam) ; \}}

Subject response. A response can be received from the keyboard after a stimulus has been presented. The example below allows a single key response. A flag is used to indicate that input has been received. Note that the code for a subject response here belongs in the stimulus window's handler function.

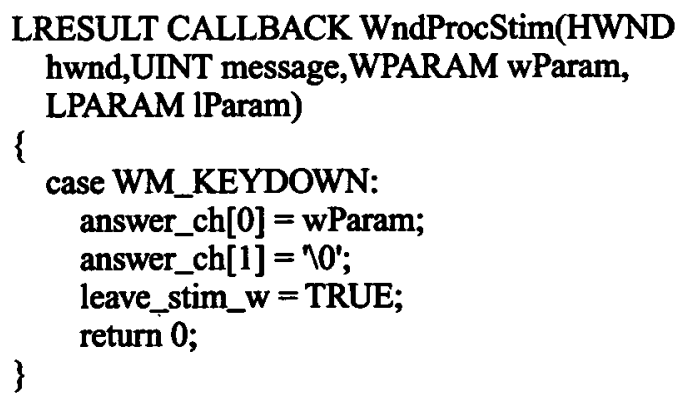

Using a separate window for text messages and input. In addition to the stimulus window, an $\mathrm{I} / \mathrm{O}$ window can be created. For the most part, the text window is initialized in the same manner as the stimulus window. If a separate window is created for receiving input, it is best to use a separate handler for it, as specified by lpfnWndProc.

To process text input, the window handler must process the WM_KEYDOWN message. Although other messages that indicate the state of the keyboard exist, WM KEYDOWN is sufficient for basic input processing.

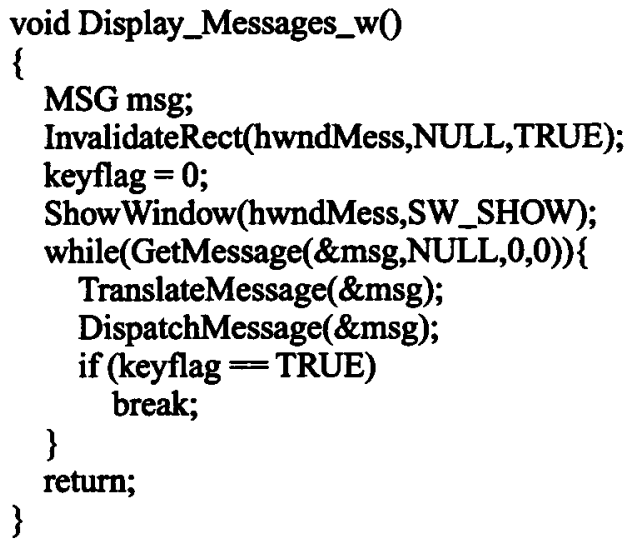




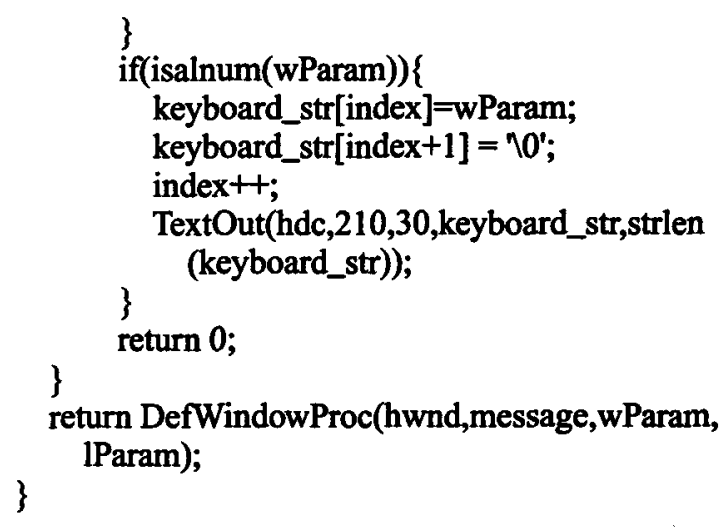

Quitting the program. Quitting the application is done by calling PostQuitMessage 0 with the return value. This API places the WM_QUIT (window message quit) message on the application's message queue, and the default behavior brings the application to a halt.

Generic program. The Appendix (www.psychonomic .org/archive) contains a working generic program that contains many of the functions necessary for stimulus generation. The program should be compiled as a Win32 windowed executable. The program contains an $\mathrm{V} / \mathrm{O}$ window for text messages and responses, and then controls three trials in which a black square moves across the screen. Following each stimulus presentation, the subject needs to enter a single keystroke as a response, after which the next trial is initiated.

\section{Other Programming Considerations}

Other considerations for programming include maintaining taskpriority level so the program is not interrupted during execution, gaining fine temporal resolution of events through computer clocks, error checking of function calls, and returning to default settings after the application terminates. Other components of trial control may also be included, such as data collection and storage and scripted input of stimulus parameters.

\section{Summary}

We have provided background to assist in the selection of computer monitors and video cards for vision research. We have also discussed operating systems, graphics implementations, and programming languages, to assist in the selection of software. The framework of a basic program for generating simple stimuli provided here can be adapted to customize software for generating and controlling stimuli at a higher level of complexity. Once developed, programs can be modified and extended with reasonable ease for continued use in vision research.

\section{AUTHOR NOTE}

Correspondence concerning this article should be addressed to D. D. Kurylo, Psychology Department, Brooklyn College, CUNY, 2900 Bedford Avenue, Brooklyn, NY 11210 (e-mail: dkurylo@brooklyn .cuny.edu).

\section{REFERENCES}

ABRASH, M. (1997). Michael Abrash's graphics programming black book (Special ed.). Albany, NY: Coriolis.

BOKHORST, F. D. (1995). Bit-plane layering for high-resolution EGA and VGA graphics on the IBM PC/XT/AT. Behavior Research Methods, Instruments, \& Computers, 27, 496-501.

DIESCH, E. (1994). A high-resolution color graphic display four-channel software tachistoscope. Behavior Research Methods, Instruments, \& Computers, 26, 331-334.

OLDS, E. S., COWEN, W. B., \& JolickUR, P. (1999). Effective color CRT calibration techniques for perception research. Journal of the Optical Society of America A, 16, 1501-1505.

PAREDES, D. R., MLLLR, K. F., \& CREeGer, C. (1990). Graphic precision: Controlling stimulus displays on IBM PC-compatible computers. Behavior Research Methods, Instruments, \& Computers, 22, 319-322.

Petzold, C. (1998). Programming Windows. Redmond, WA: Microsoft Press.

SIERRA, K., \& BATES, B. (2002). Sun certified programmer and devel. oper for Java 2 study guide (3rd ed.). Berkeley, CA: McGraw-Hill.

\section{ARCHIVED MATERIALS}

The following materials associated with this article may be accessed through the Psychonomic Society's Norms, Stimuli, and Data Archive, www.psychonomic.ong/archive.

To access these files, search the archive for this article using the journal name (Behavior Research Methods), the first author's name (Bukhari) and the publication year (2008).

FILE: Bukhari_Behavior_Research_Method_flowchart.doc; flow chart for the stimulus-generating program.

FIIE: Bukhari_Behavior_Research_Method_program_code.c; source code for the stimulus-generating program.

AUTHOR's E-MAIl ADDRESS: dkurylo@brooklyn.cuny.edu

(Manuscript received March 6, 2007; revision accepted for publication July 20,2007 .) 